\title{
Paediatric haemodynamic modelling: development and experimental validation using quantitative flow MRI
}

\author{
Parvin Mohammadyari ${ }^{1}$, Giacomo Gadda $^{2^{*}} \mathbb{B}$, Angelo Taibi ${ }^{1}$ and Josep Munuera del Cerro ${ }^{3}$
}

\begin{abstract}
Background: Congenital vascular disease is one of the leading causes of death in paediatric age. Despite the importance of paediatric haemodynamics, large investigations have been devoted to the evaluation of circulation in adults. The novelty of this study consists in the development of a well calibrated mathematical model of cardiovascular circulation in paediatric subjects. To reach the purpose, a model for adult circulation was modified and recalibrated with experimental data and literature from children to be able to calculate the flow rates and pressures in the brain and neck.
\end{abstract}

Methods: The haemodynamic model simulates the 76 main arteries, together with the main veins in brain and neck. A proper magnetic resonance imaging (MRI) dataset of 29 volunteers aged $12 \pm 5$ years (mean \pm standard deviation) was used to extract age-dependent physiological and clinical parameters such as heart rate, flow rate, vessel cross section area, and blood pressure. The computational model was calibrated using such experimental data. The paediatric and adult model results were compared.

Results: Increase of the vessels stiffness due to aging contributes to a flow rate decrease while blood pressure increases. In accordance, our simulation results show about 16\% decrease in mean pressure of internal jugular vein in paediatric rather than adult subjects. The model outcomes indicated about $88 \%$ correlation with MRI data.

Conclusions: The mathematical model simulates the paediatric head and neck blood circulation. The model provides detailed information of human haemodynamics including arterial and venous network to study both paediatric and adult blood circulation.

Keywords: Blood pressure, Cardiovascular system, Computer simulation, Hemodynamics, Pediatrics

\section{Key points}

- Aging affects the physiology of the cardiovascular system.

- Age-related physiological parameters need to be considered in a paediatric haemodynamic model.

- Our computer simulation validated by MRI at the level of head and neck provides detailed information to study haemodynamics in both paediatric and adult population.

\footnotetext{
* Correspondence: gadda@fe.infn.it

${ }^{2}$ National Institute for Nuclear Physics (INFN) - Section of Ferrara, Via

Giuseppe Saragat, 1, 44122 Ferrara, Italy

Full list of author information is available at the end of the article
}

\begin{abstract}
Background
Cardiovascular disorders are one of the most common forms of congenital defects in new-borns. The number of involved paediatric patients is lower than that of acquired cardiovascular disorders in adults; however, the mortality rate is higher [1]. Therefore, the importance of studying the paediatric vascular system is straightforward. Also, it is proven that some physiological parameters such as cardiac output $(\mathrm{CO})$, cerebral blood flow (CBF), and arterial stiffness are age-dependent $[2,3]$. The importance is emphasised by the claim: "children are not small adults" [4]. As a consequence, it is inappropriate to generalise or extrapolate data from adult to calculate the parameters and build a paediatric model $[4,5]$.
\end{abstract}


The cardiovascular system is a challenging subject of study due to the complex geometry and the high number of enrolled physiological parameters [6]. In the case of paediatric studies, the difficulty is higher because of the substantial limitations such as lack of standardising data due to patient intolerance to the clinical examinations, different physiology, and higher tissue sensitivity in comparison with adults. These barriers limit the options for choosing proper diagnostic methods [5]. Thereby, the need for more paediatric cardiovascular studies is frequently highlighted $[2,3,5]$.

The physiological parameters undergo a progressive change with age. However, by the age of $8-12$ years, the body reaches a relatively mature stage that resembles the adult body [7]. For instance, the CBF at rest increases during infancy until early childhood and then decreases about 40 to $50 \%$ until adulthood [2, 5, 8]. For this reason, it is possible to use the anatomical map and mathematical equations of the adult model as a basement in order to establish a paediatric model. However, the physiological age-dependent parameters such as vessels resistance and capacitance should be adjusted and calibrated for such paediatric age to regulate the blood flow and pressure calculated by the model. By this, we are allowed to use the same equations for the adult model with recalculated physiological parameters.

Mathematical modeling and computer simulation have been developing rapidly to study blood circulation [9]. Their benefits are not only limited to the understanding of the fluid behaviour in vessels but also for surgical planning and intervention [1]. Most of the numerical studies were dedicated to computational fluid dynamics investigation in modeling the cardiovascular and pulmonary vessel diseases while less attention has been paid to the study of the neck and brain vascular diseases $[6,10]$. Models are regulated on the basis of physics and mechanics rules to understand the influences of the involved biological factors [11-13]. Moreover, pathological cases can be simulated starting from a model previously calibrated to mimic healthy subjects [14]. Indeed, such modeling must carry on comprehensive information about the morphology of the human vessels tree that is crucial to understand how a given change of the physiological parameters can affect the results. That is our motivation to improve the anatomic map of the model.

Anatomically speaking, the internal jugular veins (IJVs) connect the superior vena cava to the venous sinus in the skull. Each of the two IJVs is conventionally subdivided into three segments (J3, J2, and J1, from the head to the heart) and its cross-sectional area increases along the same direction $[15,16]$. Due to their large cross- sectional area, IJVs are the main blood outflow pathway from the brain in the supine position [17].

Vertebral veins, internal venous plexus, and deep cervical veins are alternative pathways $[13,18]$. There are communicating pathways between external jugular veins (which collect blood from temporal branches $[15,16]$ ), IJVs, and deep cervical veins, playing an important role in improving the accuracy of the model output [18].

In terms of clinical needs, a large group of diseases which are known as different types of stenosis (like Moyamoya disease [19] and Chiari malformation [20]) can be studied by using a comprehensive mathematical model. Therefore, computational modeling can be considered as a powerful tool for clinical applications.

In this study, the mathematical model built by Gadda et al. $[11,14,21]$ is modified to investigate more precisely the human brain drainage. The model considers the cardiovascular system as an electrical circuit where blood flow rate and pressure at the $x$ anatomical level represent the electrical current and voltage, respectively. Accordingly, physiologic parameters of blood circulation are calculated $[13,14]$.

Few literature explored the internal and external cranial haemodynamics through mathematical models of the whole body circulation, and none of them relates to paediatrics. The aim of this study was to introduce a haemodynamic computational model for paediatrics. To do that, we managed a magnetic resonance imaging (MRI) dataset from normal subjects. Physiological agerelated parameters were calculated by both the dataset and literature to adjust $\mathrm{CO}, \mathrm{CBF}$, and respiratory rate for paediatrics.

\section{Methods}

\section{Patients}

Patient selection and MRI acquisition took place at the Sant Joan de Déu Hospital (Barcelona, Spain). Twentynine healthy volunteers participated in the study (Table 1 ). Subjects were chosen among all the patients for whom a brain MRI had been requested. We considered only subjects with recognised normal neck and brain blood circulation that signed the informed consent to participate as a volunteer. The study was approved by the ethical committee of the hospital.

\section{MRI protocol}

The volunteers were imaged using a 3-T scanner (Ingenia, Philips, Amsterdam, Netherlands) with a 32-channel head coil. Phase-contrast sequences were used to collect two-dimensional quantitative flow images of the neck at the vertebral level $\mathrm{C} 2 / \mathrm{C} 3$ (J3), C5/C6 (J2), and at the brain level including the Willis circle and Sylvian aqueduct, with the subject in the supine position. The common technical parameters of the sequences were as 
Table 1 Characteristic of the enrolled subjects

\begin{tabular}{lr}
\hline Demographic characteristics & 29 \\
\hline Total number of subjects & $14: 15$ \\
Sex (females:males) & $12 \pm 5$ \\
Age (years) & $2-18$ \\
Age range (years) & $43 \pm 15$ \\
Weight (kg) & $114 \pm 9$ \\
Systolic blood pressure (mmHg) & $83 \pm 16$ \\
Heart rate (beats per min) & \\
Clinical characteristics & 10 \\
Headache & 4 \\
Epilepsy & 2 \\
Cyst & 2 \\
Hearing loss & 1 \\
Down syndrome & 1 \\
Acondroplasia & 1 \\
Diplopia & 1 \\
Tremor & 1 \\
Vertigo & 1 \\
Encephalopathy (NBIA_PKAN mutation) & 1 \\
Unknown encephalopathy-genetic origin & 1 \\
Cavernoma & 1 \\
Psychosis & 1 \\
Control trauma & 1 \\
\hline Data given as absolute frequencies or mean stand & 1 \\
\hline
\end{tabular}

Data are given as absolute frequencies or mean \pm standard deviation

follows: repetition time $9.1 \mathrm{~ms}$; echo time $5.4 \mathrm{~ms}$; flip angle $15^{\circ}$; slice thickness $4 \mathrm{~mm}$; field of view $150 \mathrm{~mm} \times$ $150 \mathrm{~mm}$; reconstruction voxel size $0.6 \mathrm{~mm} \times 0.6 \mathrm{~mm}$; in-plane spatial resolution $1 \mathrm{~mm}^{3}$; temporal resolution 15 frames per cardiac cycle. Acquisitions were synchronised with the electrocardiogram of the scanned volunteer. We used phase images to extract data such as flow rate and pressure, and magnitude images to check the anatomy and improve the anatomic map of the model.

Two ranges of velocity encoding were tested in three subjects to improve the image quality and avoid aliasing effects. After this test, we chose the velocity encoding of 60 and $120 \mathrm{~cm} / \mathrm{s}$ for veins and arteries, respectively, and of $10 \mathrm{~cm} / \mathrm{s}$ to study the Sylvian aqueduct. Seven sequences were performed for each subject (Table 2). Resulting images were named by vein or artery at a specified anatomic location (Additional file 1).

Regions of interest (ROIs) were drawn manually on all the vessels on each sequence and then cross-sectional area of the vessels, blood flow, velocity, delay time, and pressure gradients were extracted by the Philips software (Philips IntelliSpace Portal, version 10). Blood flow was calculated by multiplying the mean velocity by the crosssectional area of the region of interest, while pressure
Table 2 Velocity encoding and acquisition time of the applied quantitative-flow two-dimensional phase-contrast sequences

\begin{tabular}{lrr}
\hline Imaged structures & $\begin{array}{l}\text { Velocity encoding } \\
\text { (cm/s) }\end{array}$ & \multicolumn{1}{c}{$\begin{array}{l}\text { Acquisition } \\
\text { time (s) }\end{array}$} \\
\hline $\begin{array}{l}\text { Sylvian aqueduct (cerebrospinal } \\
\text { fluid) }\end{array}$ & 10 & 97 \\
Brain arteries & 120 & 123 \\
Brain veins & 60 & 124 \\
Arteries at vertebral level C2/C3 & 120 & 125 \\
Veins at vertebral level C2/C3 & 60 & 153 \\
Arteries at vertebral level C5/C6 & 120 & 125 \\
Veins at vertebral level C5/C6 & 60 & 153 \\
\hline
\end{tabular}

gradient was calculated by using the modified Bernoulli equation $\Delta P=4\left(V_{p}^{2}\right)$, where $V_{p}$ is the peak velocity of blood [22].

After drawing the regions of interest on all the 15 frames acquired at each anatomical plane, the software calculated all the parameters. If the signal intensity was low (mostly in the temporal veins), the software did not calculate the pressure gradient. In other words, we eliminated from the study all the vessels with flow rate less than $0.05 \mathrm{~mL} / \mathrm{s}$ because of the low signal-to-noise ratio. Otherwise, resolution was enough to include the acquired data in the dataset.

\section{Mathematical modeling}

The model consists of a 0-D and 1-D algorithm [11]. In the 0-D algorithm, the intracranial autoregulation mechanisms and cerebral outflow were modeled according to the model by Ursino et al. [12, 23]. Each vessel was considered as an electric element with a given value of resistance, capacitance and conductance (Additional file 1) $[14,21,23-27]$. Equations satisfy the momentum and mass conservation laws and describe the pressure-area relationship. The model is managed by the software package MATLAB-Simulink 2017b.

\section{Adjusting the physiologic parameters}

Physiology of paediatric cardiovascular system is different from the adult and wrongly based on studies involving adults [5]. Thus, the adult model needs appropriate modifications. Heart rate (HR), systolic blood pressure (SBP), and diastolic blood pressure (DBP) in paediatric subjects are different than in adults [4]. The mean age of the volunteers enrolled in our dataset was $12 \pm 5$ years, while the measured average HR was $83 \pm 16$ beats per min (mean \pm standard deviation [SD]). Therefore, we set the duration of one cardiac cycle to $0.7 \mathrm{~s}$ (against a duration of $0.8 \mathrm{~s}$ in the adult model). The mean value of SBP (in $\mathrm{mmHg}$ ) was calculated by using the equation $\mathrm{SBP}=90+2($ Age $)$, where age is measured in years [28] 
Therefore, the SBP for our dataset was equal to $114 \pm 9$ $\mathrm{mmHg}$ (mean $\pm \mathrm{SD})$.

For what concern the mean value of DBP, we referred to literature [28-30]. From the literature we know that the respiratory rate in adults and old-children are about 15 and 20 breaths/min, respectively [30]. We tuned the respiratory rate in the model accordingly. By setting the model with these new parameters, the new simulated

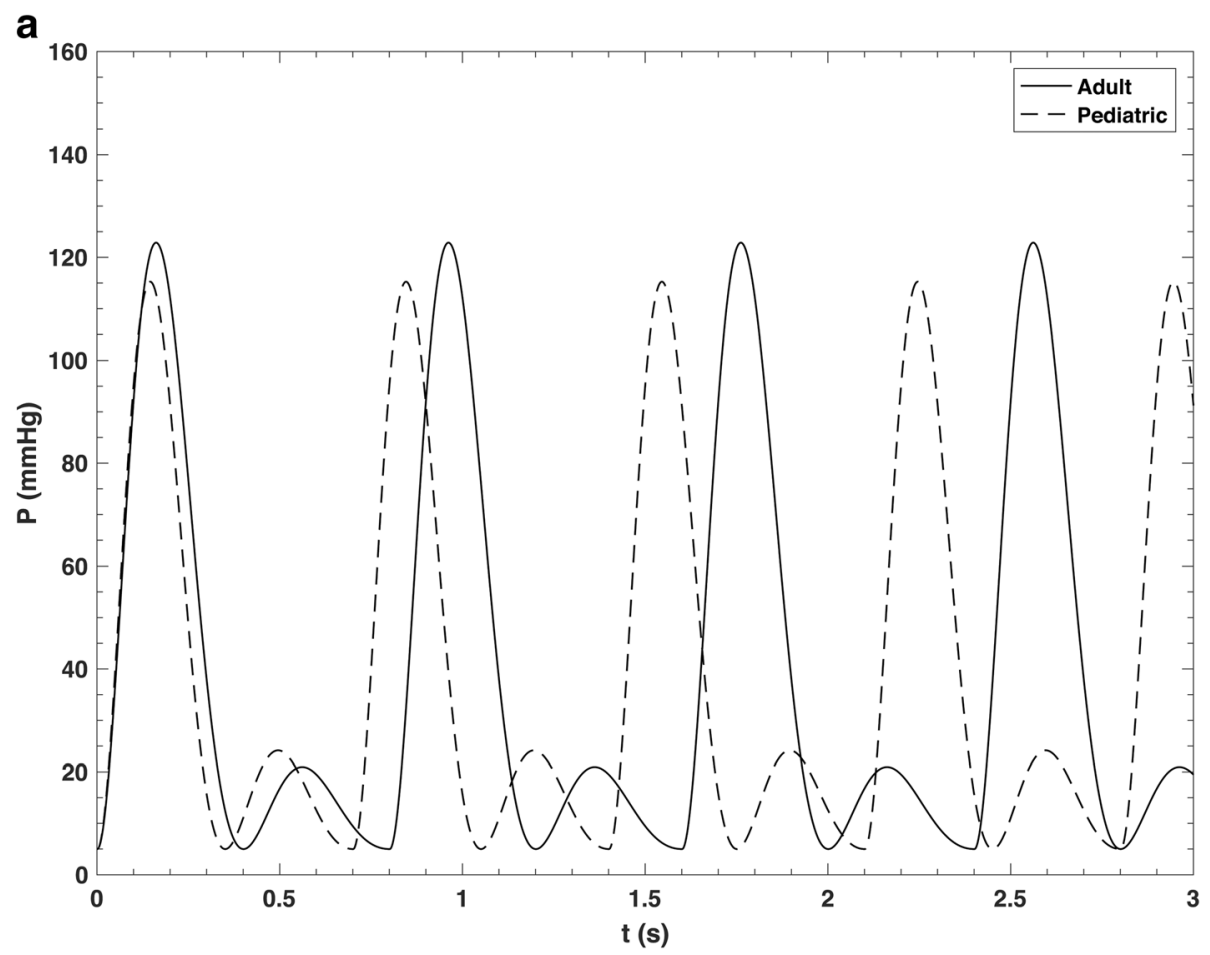

b

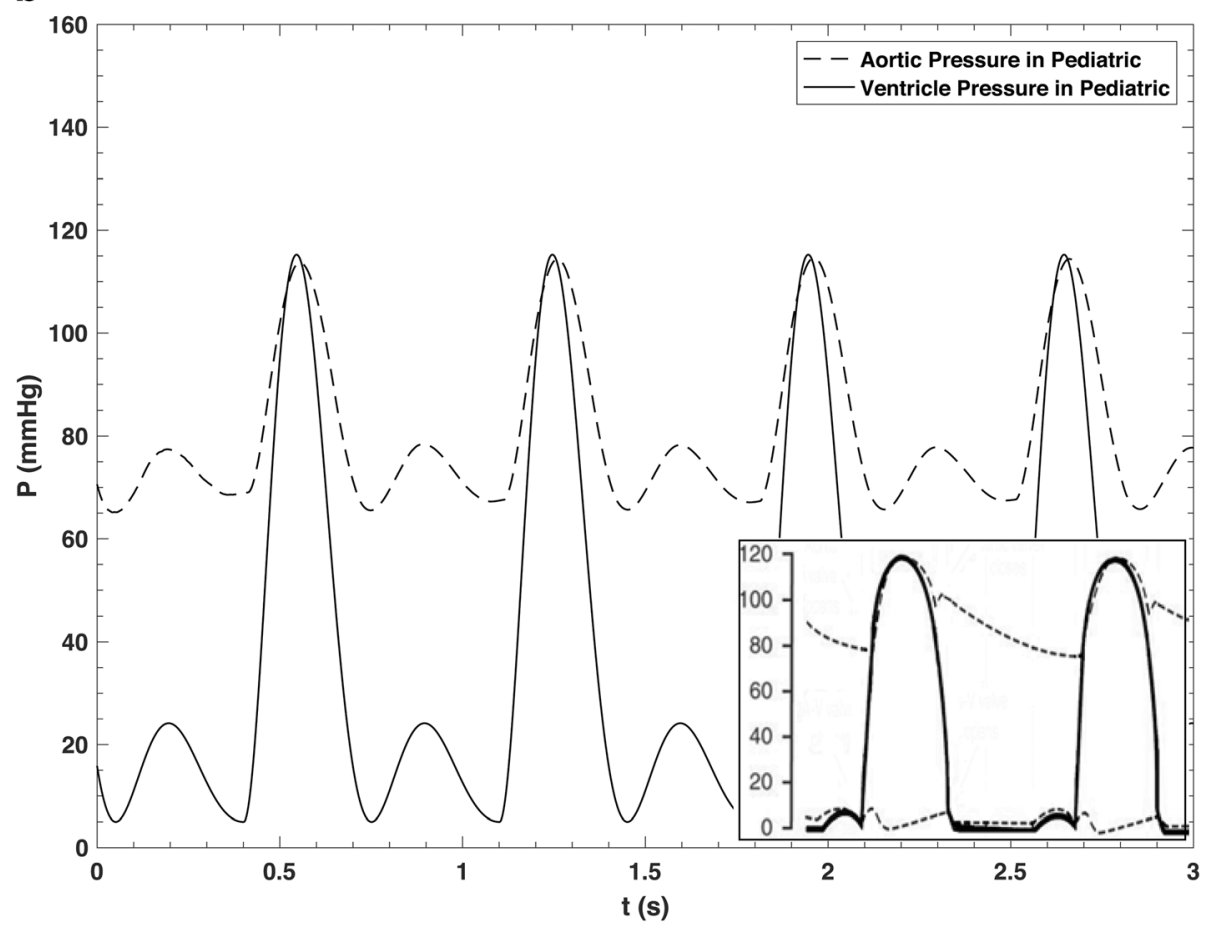

Fig. 1 The simulated cardiac pressure waveform. a Comparison of the aortic and ventricle pressure wave in paediatric. $\mathbf{b}$ Comparison of the ventricle pressure wave in paediatric and adult. The inset shows the physiological pattern 
cardiac pulse waves at the level of aorta and ventricle were representative of a paediatric cohort. In Fig. 1, such pulse waves are presented and compared with the results of the adult setting. The generated pulse wave is compared with the physiologic reference pattern for adults [24] represented in the inset of Fig. 1. The parameters were also regulated to generate the pressure waveform at the level of ascending aorta to satisfy the SBP and DBP according to the mean values from our database and the paediatric cardiopulmonary care guide [27]. More modifications were not necessary because there is no significant correlation between the mean value of systolic to diastolic ratio and age or body surface [31].

Other relevant age-related parameters to regulate the pressure and flow rate in the 1-D part of the model are total blood volume (TBV), cerebral blood volume (CBV), and cardiac volume output ratio index (CBV/CO) [2]. The $\mathrm{CO}$ is calculated by multiplying HR by the difference between end-diastolic volume and end-systolic volume [17]. Since the ascending aorta was not in the selected region of our study, we were not able to calculate the cardiac volume output ratio index. This value was taken from literature. Its mean value in infants, paediatric subjects with the same mean age of our dataset, and adults is $34.0 \%, 22.9 \%$, and $14.3 \%$, respectively $[2,32]$. The simulated flow ratio percentage of CBF to the ascending aorta flow was tuned to be about $22.9 \%$. According to literature, the paediatric TBV and CBF have the mean value of $3.4 \pm 1.2 \mathrm{~L}$ (mean $\pm \mathrm{SD}$ ) and $1101 \pm 258 \mathrm{~mL} / \mathrm{min}($ mean $\pm \mathrm{SD})(80 \mathrm{~mL}$ blood $/ 100 \mathrm{~mL}$ brain), respectively $[5,32]$. The weight of the brain is reported as $1100.7 \pm 209.6 \mathrm{~g}$ (mean \pm SD) and $1189 \pm 99 \mathrm{~g}$ (mean $\pm \mathrm{SD}$ ) in paediatric subjects and adults, respectively [2, 32]. For adults, the reported mean TBV and $\mathrm{CBF}$ are $5.6 \pm 0.3 \mathrm{~L}$ and $750(490-770) \mathrm{mL} / \mathrm{min}$ (mean and min to max range) (70 mL blood/100 $\mathrm{mL}$ of brain), respectively $[2,11,13,32]$.

\section{Adjusting the vessels anatomy map of the model}

Peripheral vessels play a considerable role in the brain and head drainage. In cases of obstructions of the main pathways, alternative routes compensate the blood outflow $[13,16,17]$. We measured the flow rate at different vessels (vessels with flow rate less than $0.05 \mathrm{~mL} / \mathrm{s}$ were eliminated from the study because of the low signal-tonoise ratio). We adjusted the anatomic map of the model to improve its ability to evaluate both physiological and pathological cases. The full list of vessels added in this work is reported in Table 3.

We worked in order to keep the proper relative magnitude among flow rates in the three segments of the IJV $[14,21,33]$. A summary of our work strategy is reported in the flowchart in Fig. 2a, which shows all the contributions used to build the paediatric model. We started
Table 3 Vessels added in the present adjustment of the mathematical model

Veins

External jugular (right and left)

Deep cervical (right and left)

External-internal jugular connection (right and left)

Internal jugular-deep cervical connection (right and left)

Arteries

Internal carotid (right and left)

Middle cerebral (right and left)

Anterior cerebral (right and left)

Posterior cerebral (right and left)

Basilar

Anterior communicating

Posterior communicating (right and left)

External carotid (right and left)

Ophthalmic (right and left)

Facial (right and left)

The vascular structure already included in the model were the following: internal jugular veins, anastomotic connections, azygos plexus, cerebrospinal inflow, and outflow pathways (Fig. 2b) $[11,14,21]$; main arterial tree of the human body outside the braincase (Fig. 2b, arteries 1 to 55$)[25,26]$

from mathematical models previously validated and published by our group to have a solid basis from the mathematical point of view. Beside, we used both literature and data acquisition to collect all the parameters we need to switch from an adult to a paediatric model, by a proper tuning of the parameters of the mathematical equations. The adjusted hydraulic map of the vascular system is depicted in Fig. 2b.

\section{Results}

\section{Arterial adjustments}

The pressure pulse wave of Fig. 1, together with the contribution of the respiratory pulse are the input to the model. As shown in Fig. 3, the simulated pressure in the ascending aorta was $114 / 65 \mathrm{mmHg}$, in accordance with the paediatric cardiopulmonary care guide [27]. Figure 3 also reports the pressure pulse in the intracranial artery, extracranial artery, vertebral artery, and facial arteries calculated by the model.

The backward wave from ascending aorta to heart generate the late systolic or early diastolic pressure peak $[18,24]$. This late pulse can be seen in Fig. 3. Moreover, the blood flows from the heart to the vessels and is divided among many branches. This must correspond to a change in the venous pressure. We can see from Fig. 3 that the model properly simulates both decrease in pressure amplitudes and mean values in the following order: ascending aorta $>$ intracranial artery $>$ extracranial artery $>$ facial arteries $[13,16,24]$. 

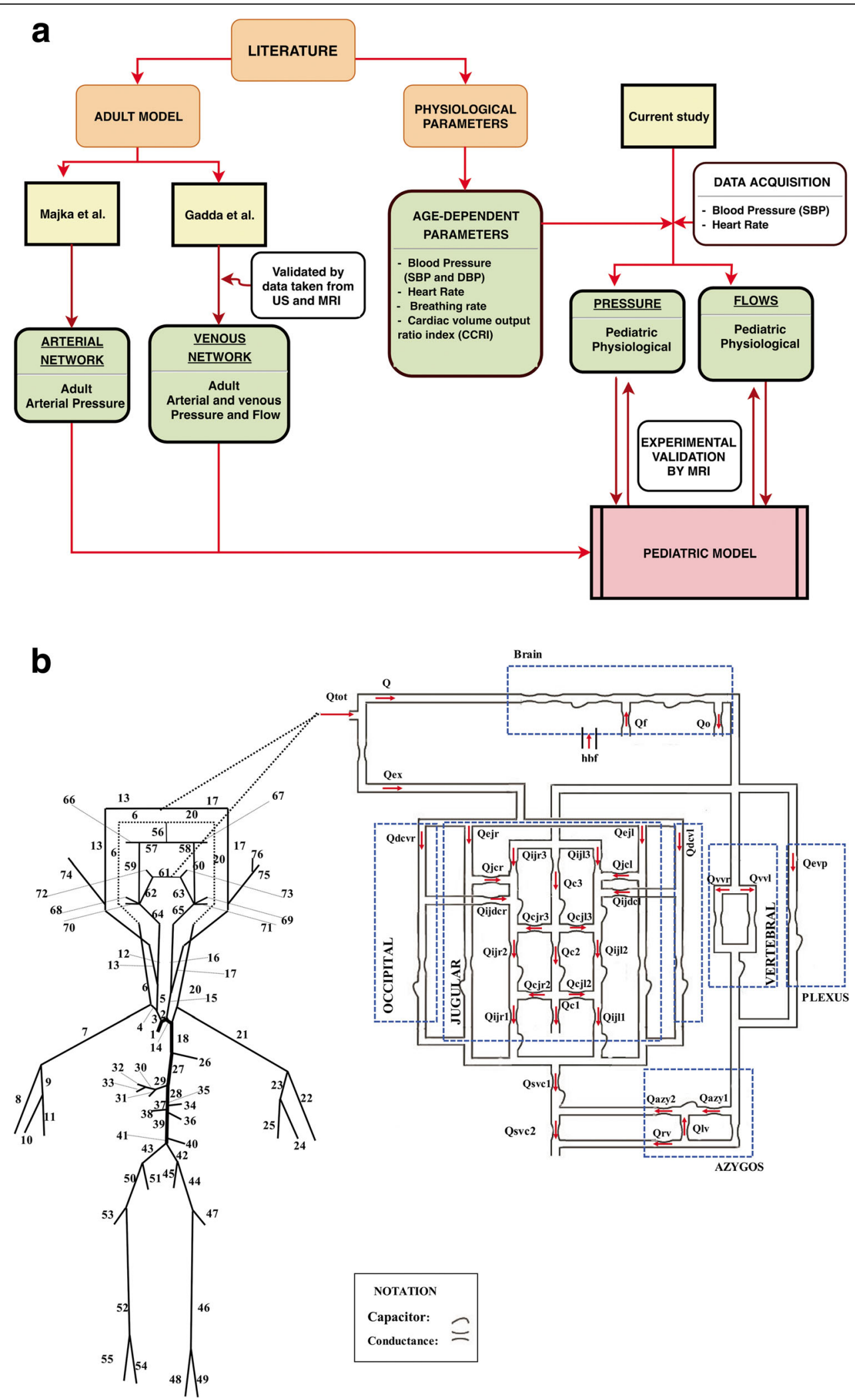

Fig. 2 (See legend on next page.) 
(See figure on previous page.)

Fig. 2 a Flowchart of all the contributions used to build the paediatric model. $\mathbf{b}$ Scheme of the modified haemodynamic model with all the new anatomical adjustments. hbf Cerebrospinal fluid possibly injected into or subtracted from the cranial cavity, azy 1 Distal azygos, azy2 Proximal azygos, c1 Lower segment of the collateral network, c2 Middle segment of the collateral network, c3 Upper segment of the collateral network, cjl2 Lower anastomotic connection (left side), cjl3 Upper anastomotic connection (left side), cjr2 Upper anastomotic connection (right side), cjr3 Upper anastomotic connection (right side), dcvl Left deep cervical vein, dcvr Right deep cervical vein, ejl Left external jugular vein, ejr Right external jugular vein, evp External venous plexus, ex Extracerebral, $Q_{f}$ Cerebrospinal fluid formation rate, ijdcl Internal jugular-deep cervical connection (left side), ijdcr Internal jugular-deep cervical connection (right side), ij/1 Lower segment of the left internal jugular vein, ij/2 Middle segment of the left internal jugular vein, ij/3 Upper segment of the left internal jugular vein, ijr Lower segment of the right internal jugular vein, ijr2 Middle segment of the right internal jugular vein, ijr3 Upper segment of the right internal jugular vein, jcl External-internal jugular connection (left side), jcr External-internal jugular connection (right side), IV Lumbar vein, $Q_{0}$ Cerebrospinal fluid outflow rate, $r v$ Renal vein, svcl Superior vena cava (upper segment), svc2 Superior vena cava (lower segment), vvl Left vertebral vein, $v$ rr Right vertebral vein

Figure 4 compares the simulated intracranial artery pressure and venous sinus pressure in paediatric subjects and adults. We see that the mean pressure values were less in lower age. Indeed, the mean values of intracranial artery pressure are $5.6 \pm 0.2$ (mean \pm SD) and $8.3 \pm 0.8$ $\mathrm{mmHg}$ (mean $\pm \mathrm{SD}$ ), while the mean values of venous sinus pressure are $4.9 \pm 0.6$ and $5.9 \pm 1.0 \mathrm{mmHg}$ in paediatric subjects and adults, respectively. Moreover, we see that intracranial artery pressure was lower than venous sinus pressure. Hence, the simulated intracranial artery pressure and venous sinus pressure values were in agreement with the literature. The normal range of intracranial artery pressure was variable between 3 and 7 $\mathrm{mmHg}$ for paediatric subjects and less than 10-15 mmHg for adults [34, 35].

\section{Venous adjustments}

The venous part of the model was adjusted to simulate the main blood pathways from the brain. In the majority of individuals, IJVs are the main pathway and the flow at each segment (J1, J2, and J3) is more than that of vertebral veins or internal venous plexus flow [14, 34]. In this work, we refer to the flow rate in VV and DCV as summation of the left and right veins due to the low value of them. The intracranial flow rate is calculated by the summation of ICA left and right and basilar arteries [2]. Moreover, total blood flow is the flow rate at the level of neck arteries that supplies the intracranial and extracranial subsystems $[14,21]$. Figure 5 shows the amount of the simulated flow rates at rest and makes a comparison between paediatric and adult flow rates.

There was good agreement between model response and paediatric MRI flow data. Moreover, flow rates were higher in paediatric rather than adult subjects, as expected.

Figure $6 \mathrm{a}-\mathrm{f}$ shows how the model simulates the pressure and flow rate both in paediatric (dashed line) and adult (solid line) setting in IJV and vertebral veins. Figure $6 \mathrm{~g}-\mathrm{i}$ compares the simulated flow waveforms

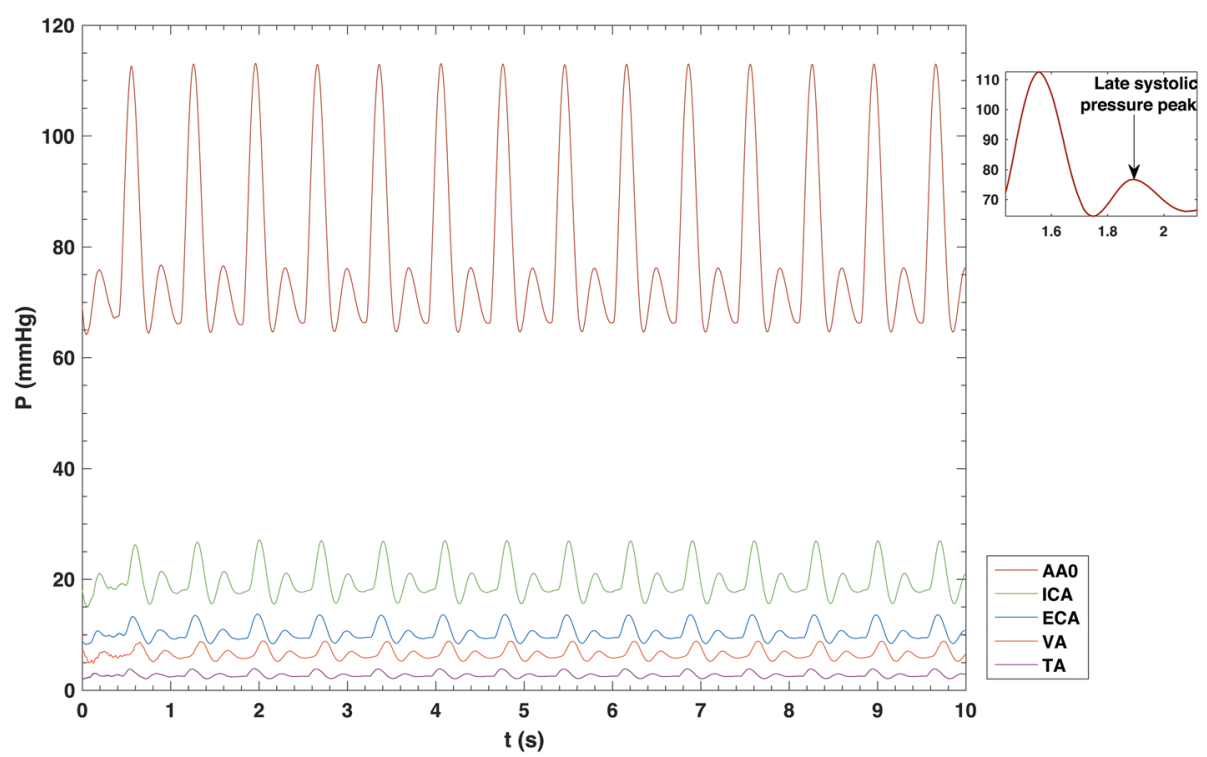

Fig. 3 Cardiac and arterial pressure waveforms. The inset shows the zoom of one cardiac cycle. AAo Ascending aorta, ECA Extracranial artery, ICA Intracranial artery, FA Facial artery, VA Vertebral artery 

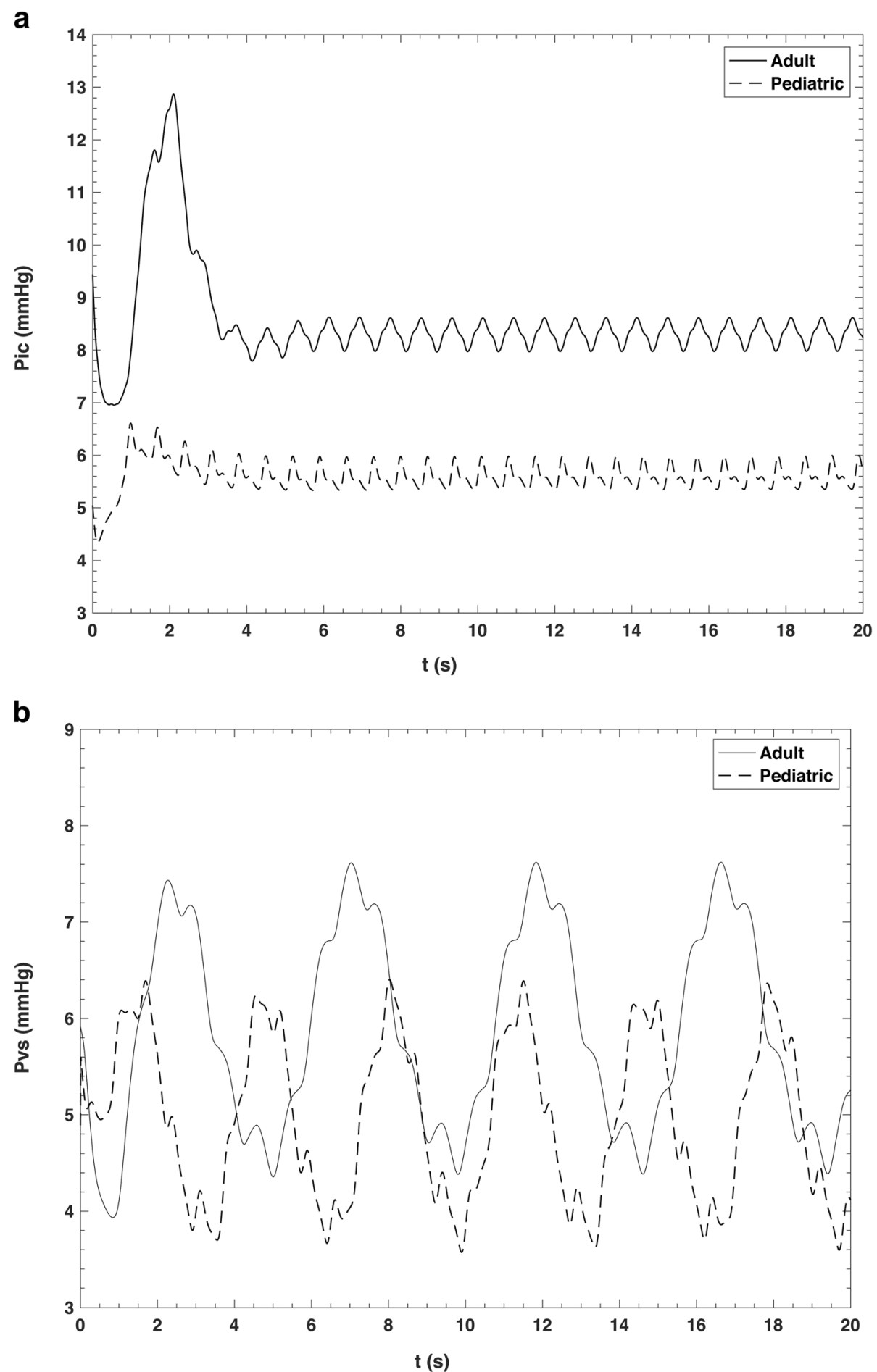

Fig. 4 Comparison between $(\mathbf{a})$ intracranial $\left(P_{\mathrm{ic}}\right)$ and $(\mathbf{b})$ venous sinus $\left(P_{\mathrm{vs}}\right)$ pulse in paediatric (dashed line) and adult (solid line)

in IJV and VV against PC-MRI data. In this figure, the simulation of respiratory rate and so the effects on modeled flow rate was eliminated in order to make a comparison with phase-contrast data of one cardiac cycle. In order to quantify the agreement shown in Fig. 6g-i, we calculated the time-averaged difference between simulated (dashed line) and measured (dotted line) flow rates, during the reported cardiac cycle, for left J3, left J2, and vertebral vein. Such time-averaged difference was $1.1 \pm 0.3 \mathrm{~mL} / \mathrm{s}$ 


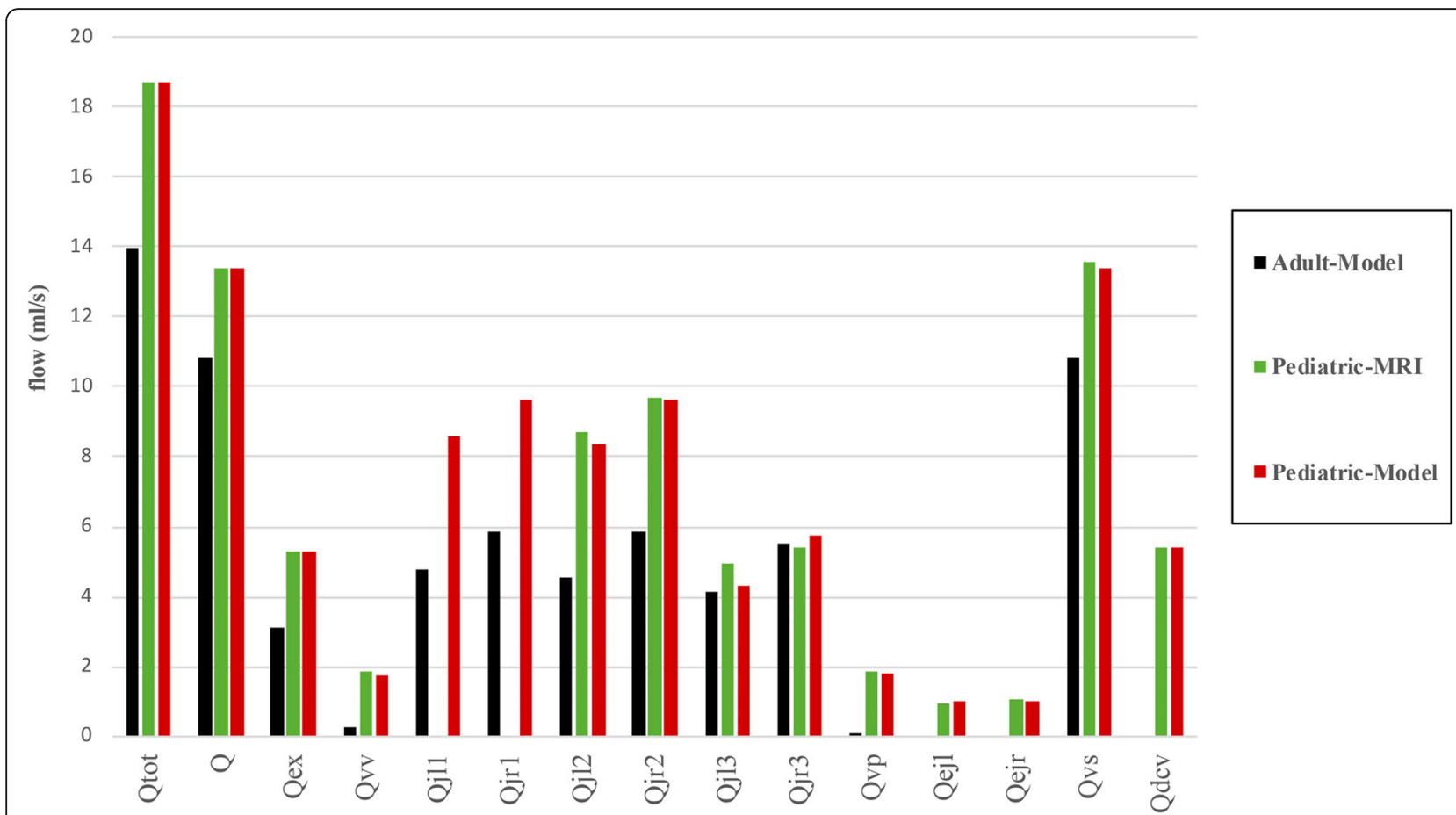

Fig. 5 Comparison of simulated paediatric and adult flow rates, and paediatric experimental data. Q Cerebral blood flow, dcv Deep cervical vein, ejl Left external jugular vein, ejr Right external jugular vein, $Q_{e x}$ Extracerebral blood flow, j/1 Lower segment of the left jugular vein, j/2 Middle segment of the left jugular vein, j/3 Upper segment of the left jugular vein, jr1 Lower segment of the right jugular vein, jr2 Middle segment of the right jugular vein, jr3 Upper segment of the right jugular vein, $Q_{\text {tot }}$ Blood flow to the head, vp Venous plexus, vs Venous sinus, vv Vertebral vein

$($ mean $\pm \mathrm{SD}), 3.7 \pm 0.1 \mathrm{~mL} / \mathrm{s}($ mean $\pm \mathrm{SD})$, and $0.3 \pm$ $0.2 \mathrm{~mL} / \mathrm{s}$ (mean $\pm \mathrm{SD})$, respectively.

\section{Discussion}

Based on a well-developed mathematical model for the simulation of blood circulation in adult humans [11], the present model has been tuned with respect to paediatric physiological parameters (average age 12 years, supine position).

In 2017, Cattermole et al. [35] reported a measured values of ascending aorta pressure of $80.7 \mathrm{mmHg}$ (range 65.3 to $103.7 \mathrm{mmHg}$ ) in the same age range as this study (see Fig. 3). Our simulated pressure in the intracranial artery $(22 \pm 4 \mathrm{mmHg}$, mean $\pm \mathrm{SD}$, age range 2 to 18 years old) follows the trend of the data reported in literature (age range 20 to 60 years old and older), where blood pressure in intracranial artery increases with age. Indeed, on 2006 Hirata et al. [36] investigated blood velocity and pressure in intracranial artery and reported a mean value of $29.3 \pm 6.4 \mathrm{mmHg}$ (mean $\pm \mathrm{SD}$ ) for the age range from 20 to 40 years, $35.9 \pm 8.0 \mathrm{mmHg}$ (mean $\pm \mathrm{SD}$ ) from 40 to 60 years and higher values for higher ages.

The CBV increases rapidly from early childhood to preschool age up to 6 years, and then continuously decreases till adulthood $[2,8]$. Moses et al. [8] reported a normalised different value of $10.6 \pm 5.2 \mathrm{~mL} / 100 \mathrm{~mL} / \mathrm{min}$ between the mean age of 12 years and adults (mean age of 22 years) [8]. Wu et al. [2] reported a blood flow reduction rate of about $48 \%$ from the age of 12 to 40 years [2]. According to Fig. 5, the average inflow toward head and brain are $18.7 \mathrm{~mL} / \mathrm{s}$ and $13.4 \mathrm{~mL} / \mathrm{s}$ that is a $34 \%$ and $24 \%$ augmentation in paediatric rather than adult subjects. These values are within the range of previous reports $[2,5,8]$.

Additionally, we can see from Fig. 5 the asymmetric behaviour of the cerebral venous drainage. Indeed, the right side flow rate is about $10 \%$ more than the left, as reported by the literature [14, 33, 34, 37].

The mean value of central venous pressure and EJV pressure in adults were studied by Leonard et al. [37]. They stated that mean EJV pressure is slightly higher than IJV pressure. They measured $8.3 \pm 2.6 \mathrm{mmHg}$ $($ mean $\pm \mathrm{SD})$ for IJV and $8.6 \pm 2.8 \mathrm{mmHg}($ mean $\pm \mathrm{SD})$ for EJV in adults with a mean age of 66 years. As depicted in Fig. 6, mean IJV pressure in all three sections and vertebral vein pressure were $4.7 \pm 0.8 \mathrm{mmHg}$ (mean $\pm \mathrm{SD}$ ) and $4.8 \pm 0.7 \mathrm{mmHg}$ (mean \pm SD) in paediatric subjects, and $5.6 \pm 1.1 \mathrm{mmHg}$ (mean $\pm \mathrm{SD}$ ) and $5.8 \pm$ $0.7 \mathrm{mmHg}$ (mean $\pm \mathrm{SD}$ ) in adults, respectively, showing that the model results are quite satisfactory. However, 


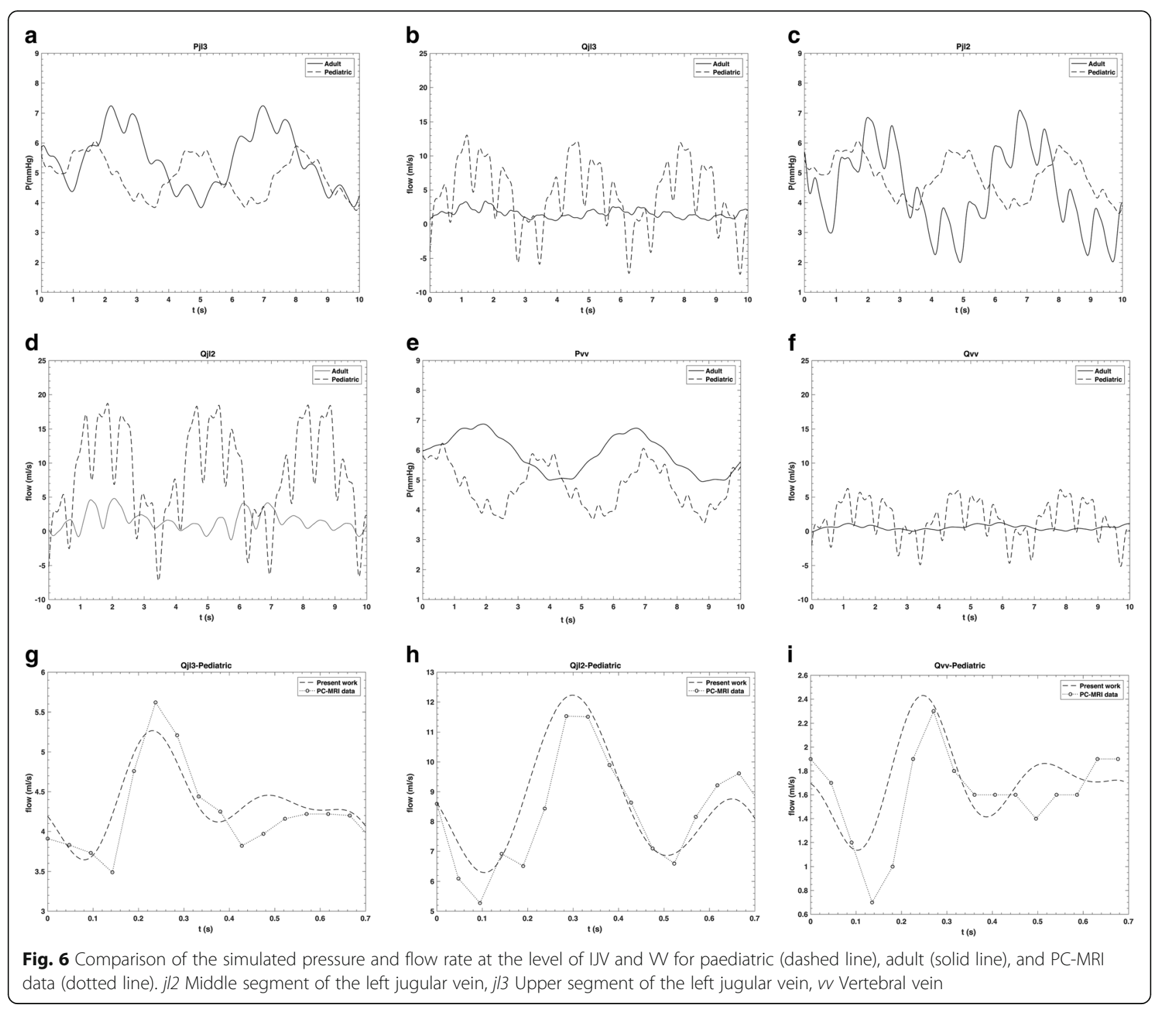

there is the need of more paediatric measurement at the IJV level and peripheral veins in order to produce a model with more details.

Results reported in Fig. 6a-f were satisfactory because the simulated pressure values were higher in adult than paediatric subjects, while flow rates were lower $[2,8]$.

Figure $6 \mathrm{~g}-\mathrm{i}$ represents a comparison between the simulated and measured flow rates in J3, J2, and vertebral vein as an example of the accuracy of our paediatric model. The correlation value between the simulated and PC-MRI data was $88 \%$. The positive value of all the calculated time-averaged differences between simulated and measured flow rates means that, in the investigated anatomical regions, the mathematical model slightly overestimates the flow rate. The low SDs are index of good agreement between simulated and measured flow rates. As previously reported in literature [38], there is interest in properly simulate flow rate and pressure waveforms to discriminate between physiologic and pathologic subjects. The present model is able to simulate such waveforms for a paediatric setting.

Our model is a suitable computational tool to study the paediatric and adult haemodynamics. In a previous paper, Gadda et al. showed the ability of the model we used to simulate healthy and pathological cases [11, 14, 21]. Here, the model is expanded and adjusted to a paediatric setting by keeping the previous abilities. Therefore, following the pathway of Gadda et al. [14], a further step will be to collect MRI datasets from paediatric subjects with different kind of vascular diseases (i.e., vessel occlusions at different levels of the vascular tree), and tune the paediatric model to properly simulate blood flows and pressure in those 
new pathological settings. For our work, the phasecontrast technique is important to assess the contribution of peripheral vessels in the human brain drainage. Indeed, PC-MRI is able to show that the external jugular, deep cervical and epidural veins play a crucial role. From this evidence, we decided to extend the anatomic map of the model from the work by Gadda et al. [11] to the version presented here. Moreover, in future work, we want to take advantage of our data from Sylvian aqueduct to improve the cerebrospinal fluid circulation related parameters and brain part of the model.

Further discussions are needed to assess the opportunity to use other MRI techniques for these purposes. We are currently going to use fourdimensional flow sequences. Such update will allow new measurements to further refine and validate the model. However, four-dimensional flow protocols are highly time-consuming to study blood flows at the neck level because of the volume involved. This protocol will extend the examination time by about 20 min, probably not acceptable for the paediatric tolerance and the review board.

Our study has limitations. To retrieve flow measurements from phase contrast imaging is sometimes not easy in small vessels, and even more difficult in veins due to the lower yields of the parameters in paediatric subjects. The patient selection protocol was affected by limitations that decrease the number of cases. Subjects were chosen among patients with brain MRI prescription. We considered two conditions to select a patient for the study: normal neck and brain blood circulation, and no sedation during MRI due to the influence of the anaesthesia medication on the brain flows [39].

According to Doepp et al. [15] there are two main categories of human blood drainage: jugular drainage (75\% of the population) and non-jugular drainage ( $25 \%$ of the population). Our dataset is in perfect agreement, showing a subset of jugular drainers equal to $74 \%$ (26\% nonjugular). Nevertheless, we decided to not separate the two subgroups in this study, focusing on the problem to tune an "adult based" model to paediatrics. Moreover, paediatric subjects are categorised in three subgroups: infants, preschool and old-children while there is no such a categorisation for adults. This implies a poor accuracy when we want to compare paediatric and adult subjects because the value of biofactors changes with age constantly. Insufficiency in the number of experimental studies and age categorisation may have affected the quality of numerical studies and modeling.

In conclusion, we modeled for the first time the paediatric haemodynamic circulation by using a parameter model consisting of a 0-D and 1-D algorithm. The previous well-described model [21] was adjusted in accordance to the differences between paediatric and adult subjects. The model was able to compute blood pressures and flow rates in accordance to the collected phasecontrast MRI data and literature. Moreover, it has the potential to simulate pathological conditions due to aging.

\section{Supplementary information}

Supplementary information accompanies this paper at https://doi.org/10. 1186/s41747-020-0146-X.

Additional file 1. Supplemental material to "paediatric haemodynamic modelling: development and experimental validation using quantitative flow MRI

\section{Abbreviations}

CBF: Cerebral blood flow; CBV: Cerebral blood volume; CO: Cardiac output; DBP: Diastolic blood pressure; DCV: Deep cervical vein; EJV: External jugular vein; HR: Heart rate; IJV: Internal jugular vein; J1: Internal jugular vein, segment 1; J2: Internal jugular vein, segment 2; J3: Internal jugular vein, segment 3; SBP: Systolic blood pressure; SD: Standard deviation; TBV: Total blood volume

\section{Authors' contributions}

GG, AT, and JM contributed to the conceptualisation, project administration, and supervision of the study. GG, PM, and JM curated the data. GG and PM contributed to the formal analysis and wrote the original draft. GG, PM, AT, and $\mathrm{JM}$ contributed to the investigation, methodology, and validation, and helped write, review, and edit the manuscript. PM was responsible for the software. GG contributed to the visualisation. All authors read and approved the final manuscript.

\section{Funding}

The authors state that this work has not received any funding.

\section{Availability of data and materials}

The datasets used and/or analysed during the current study are available from the corresponding author on reasonable request.

Ethics approval and consent to participate

Institutional Review Board approval was obtained.

Written informed consent was obtained from all subjects (patients) in this study

\section{Consent for publication}

Written informed consent was obtained from all subjects (patients) in this study.

\section{Competing interests}

The authors declare that they have no competing interests.

\section{Author details}

${ }^{1}$ Department of Physics and Earth Sciences, University of Ferrara, Via Giuseppe Saragat, 1, 44122 Ferrara, Italy. ${ }^{2}$ National Institute for Nuclear Physics (INFN) - Section of Ferrara, Via Giuseppe Saragat, 1, 44122 Ferrara, Italy. ${ }^{3}$ Department of Diagnostic Imaging, Fundació Sant Joan de Déu, Passeig de Sant Joan de Déu, 2, 08950 Esplugues de Llobregat, Barcelona, Spain

Received: 23 September 2019 Accepted: 24 January 2020 Published online: 16 March 2020

\section{References}

1. Slesnick TC (2017) Role of computational modelling in planning and executing interventional procedures for congenital heart disease. Can J Cardiol 33:1159-1170. https://doi.org/10.1016/j.cjca.2017.05.024

2. Wu C, Honarmand AR, Schnell S et al (2016) Age-related changes of normal cerebral and cardiac blood flow in children and adults aged 7 months to 61 years. J Am Heart Assoc 5. https://doi.org/10.1161/JAHA.115.002657 
3. Shirwany NA, Zou MH (2010) Arterial stiffness: a brief review. Acta Pharmacol Sin 31:1267-1276. https://doi.org/10.1038/aps.2010.123

4. Macfarlane $F$ (2005) Paediatric anatomy and physiology and the basic of paediatric anaesthesia. World Anaesthesia Tutorial of the week. AnaesthesiaUK. Available via https://www.AnaesthesiaUK.com/ WorldAnaesthesia. Accessed 12 Oct 2015

5. Figaji AA (2017) Anatomical and physiological differences between children and adults relevant to traumatic brain injury and the implications for clinical assessment and care. Front Neurol 8:685. https://doi.org/10.3389/fneur.2017.00685

6. Moghadam ME, Vignon-Clementel IE, Figliola R, Marsden AL (2013) A modular numerical method for implicit 0D/3D coupling in cardiovascular finite element simulations. J Comput Phys 244:63-79. https://doi.org/10. 1016/j.jcp.2012.07.035

7. Takahashi T, Shirane R, Sato S, Yoshimoto T (1999) Developmental changes of cerebral blood flow and oxygen metabolism in children. AJNR Am J Neuroradiol 20:917-922

8. Moses P, Hernandez LM, Orient E (2014) Age-related differences in cerebral blood flow underlie the BOLD fMRI signal in childhood. Front Psychol 5:300. https://doi.org/10.3389/fpsyg.2014.00300

9. Toro EF (2016) Brain venous haemodynamics, neurological diseases and mathematical modelling. A review. Appl Math Comput 272:542-579. https:// doi.org/10.1016/j.amc.2015.06.066

10. Qureshi MU, Vaughan GD, Sainsbury C et al (2014) Numerical simulation of blood flow and pressure drop in the pulmonary arterial and venous circulation. Biomech Model Mechanobiol 13:1137-1154. https://doi.org/10. 1007/s10237-014-0563-y

11. Gadda G, Majka M, Zieliński P, Gambaccini M, Taibi A (2018) A multiscale model for the simulation of cerebral and extracerebral blood flows and pressures in humans. Eur J Appl Physiol 118:2443-2454. https://doi.org/10. 1007/s00421-018-3971-3

12. Ursino M, Lodi CA (1997) A simple mathematical model of the interaction between intracranial pressure and cerebral hemodynamics. J Appl Physiol (1985) 82:1256-1269. https://doi.org/10.1152/jappl.1997.82.4.1256

13. Gisolf J, van Lieshout JJ, van Heusden K, Pott F, Stok WJ, Karemaker JM (2004) Human cerebral venous outflow pathway depends on posture and central venous pressure. J Physiol 560:317-327. https://doi.org/10.1113/ jphysiol.2004.070409

14. Gadda G, Taibi A, Sisini F et al (2016) Validation of a hemodynamic model for the study of the cerebral venous outflow system using MR imaging and echo-color Doppler data. AJNR Am J Neuroradiol 37:2100-2109. https://doi. org/10.3174/ajnr.A4860

15. Doepp F, Schreiber SJ, von Münster T, Rademacher J, Klingebiel R, Valdueza JM (2004) How does the blood leave the brain? A systematic ultrasound analysis of cerebral venous drainage patterns. Neuroradiology 46:565-570. https://doi.org/10.1007/s00234-004-1213-3

16. Zamboni P, Morovic S, Menegatti E, Viselner G, Nicolaides AN (2011) Screening for chronic cerebrospinal venous insufficiency (CCSVI) using ultrasound--recommendations for a protocol. Int Angiol 30:571-597

17. Zamboni P, Menegatti E, Occhionorelli S, Salvi F (2013) The controversy on chronic cerebrospinal venous insufficiency. Veins and Lymphatics 2:e14. https://doi.org/10.4081/vl.2013.e14

18. Tobinick E, Vega CP (2006) The cerebrospinal venous system: anatomy, physiology, and clinical implications. MedGenMed 8:53

19. Law-ye B, Saliou G, Toulgoat F et al (2016) Early-onset stroke with moyamoyalike syndrome and extraneurological signs: a first reported paediatric series. Eur Radiol 26:2853-2862. https://doi.org/10.1007/s00330-015-4119-z

20. Manto M, Huisman T (eds) (2018) The cerebellum: disorders and treatment. Elsevier

21. Gadda G, Taibi A, Sisini F, Gambaccini M, Zamboni P, Ursino M (2015) A new hemodynamic model for the study of cerebral venous outflow. Am J Physiol Heart Circ Physiol 308:H217-H231. https://doi.org/10.1152/ajpheart.00469.2014

22. Caruthers SD, Lin SJ, Brown P et al (2003) Practical value of cardiac magnetic resonance imaging for clinical quantification of aortic valve stenosis: comparison with echocardiography. Circulation 108:2236-2243. https://doi.org/10.1161/01.CIR.0000095268.47282.A1

23. Ursino M, Ter Minassian A, Lodi CA, Beydon L (2000) Cerebral hemodynamics during arterial and $\mathrm{CO}(2)$ pressure changes: in vivo prediction by a mathematical model. Am J Physiol Heart Circ Physiol 279: H2439-H2455. https://doi.org/10.1152/ajpheart.2000.279.5.H2439

24. Guyton AC (1991) The veins and their functions. In: Textbook of medical physiology, 8th edn. Saunders, Philadelphia
25. Majka M, Gadda G, Taibi A, Gałązka M, Zieliński P (2017) Earliest effects of sudden occlusions on pressure profiles in selected locations of the human systemic arterial system. Phys Rev E 95:032414. https://doi.org/10.1103/PhysRevE.95.032414

26. Majka M, Gadda G, Taibi A, Gałąka M, Zieliński P (2017) Protective properties of the arterial system against peripherally generated waves. Math Biosci 286:16-21. https://doi.org/10.1016/j.mbs.2017.01.007

27. Larrabide I, Blanco PJ, Urquiza SA et al (2012) HeMoLab - Hemodynamics Modelling Laboratory: an application for modelling the human cardiovascular system. Comput Biol Med 42:993-1004. https://doi.org/10. 1016/j.compbiomed.2012.07.011

28. Moses S (2019) Pediatric vital signs, vital signs in children. In: Cardiovascular medicine book. https://fpnotebook.com/cv/exam/pdtrcvtlsgns.htm. Accessed 13 May 2019

29. Boville B, Caulette Young L (2015) ECCE Quick guide to pediatric cardiopulmonary care. Edwards, California

30. Butterworth JF, Mackey DC, Wasnick JD (2013) Morgan \& Mikhail's Clinical Anesthesiology. McGraw-Hill Education, New York

31. Sarnari R, Kamal RY, Friedberg MK, Silverman NH (2009) Doppler assessment of the ratio of the systolic to diastolic duration in normal children: relation to heart rate, age and body surface area. J Am Soc Echocardiogr 22:928932. https://doi.org/10.1016/j.echo.2009.05.004

32. Xing CY, Tarumi T, Liu J et al (2017) Distribution of cardiac output to the brain across the adult lifespan. J Cereb Blood Flow Metab 37:2848-2856. https://doi.org/10.1177/0271678X16676826

33. Stover JF, Stocker R (2011) Intensive care treatment options of elevated intracranial pressure following severe traumatic brain injury. In: Oestern $\mathrm{HJ}$, Trentz O, Uraunes S (eds) Head, thoracic, abdominal, and vascular injuries, cap. 2. Springer-Verlag Berlin Heidelberg

34. Wilson MH (2016) Monro-Kellie 2.0: The dynamic vascular and venous pathophysiological components of intracranial pressure. J Cereb Blood Flow Metab 36:1338-1350. https://doi.org/10.1177/0271678X16648711

35. Cattermole GN, Leung PY, Ho GY et al (2017) The normal ranges of cardiovascular parameters measured using the ultrasonic cardiac output monitor. Physiol Rep 5:e13195. https://doi.org/10.14814/phy2.13195

36. Hirata K, Yaginuma T, O'Rourke MF, Kawakami M (2006) Age-related changes in carotid artery flow and pressure pulses: possible implications for cerebral microvascular disease. Stroke 37:2552-2556. https://doi.org/10.1161/ 01.STR.0000242289.20381.f4

37. Leonard AD, Allsager CM, Parker JL, Swami A, Thompson JP (2008) Comparison of central venous and external jugular venous pressures during repair of proximal femoral fracture. Br J Anaesth 101:166-170. https://doi. org/10.1093/bja/aen125

38. Müller LO, Toro EF (2014) Enhanced global mathematical model for studying cerebral venous blood flow. J Biomech 47:3361-3372. https://doi. org/10.1016/j.jbiomech.2014.08.005

39. Gribkov AV, Boiarinov GA, Bolonichev AM, Grigor'eva W (2001) Changes in cerebral blood flow, intracranial and cerebral perfusion pressure in neurosurgical patients during anesthesia induced with diprivan. Anesteziol Reanimatol 2:43-45

\section{Publisher's Note}

Springer Nature remains neutral with regard to jurisdictional claims in published maps and institutional affiliations.

\section{Submit your manuscript to a SpringerOpen ${ }^{\circ}$ journal and benefit from:}

- Convenient online submission

- Rigorous peer review

- Open access: articles freely available online

- High visibility within the field

- Retaining the copyright to your article

Submit your next manuscript at $>$ springeropen.com 